\title{
INFLUENCE OF PROCESS PARAMETERS ON THE WELD QUALITY OF DISSIMILAR CU-AL MAGNETIC PULSE WELDED SHEETS
}

\author{
W. Demonie ${ }^{1}$, K. Faes ${ }^{2}$ and W. De Waele ${ }^{1}$ \\ ${ }^{1}$ Ghent University, Faculty of Engineering and Architecture, Laboratory Soete, Belgium \\ ${ }^{2}$ Belgian Welding Institute, Laboratory Soete, Belgium
}

\begin{abstract}
Little is known about the influence of the main geometrical parameters (overlap, stand-off and free length) and the energy input on welds formed by electromagnetic pulse welding. The Taguchi experimental design method was applied for determining the underlying relations for dissimilar sheet welding of copper and aluminium. The weld quality was evaluated based on four output parameters: the weld length, the size of the interfacial layer, the lap shear strength and the thickness reduction of the flyer sheet. The influence of the overlap and free length showed to be non-negligible; this in contrast to the small amount of attention these parameters receive in other publications.
\end{abstract}

Keywords: magnetic pulse welding; Taguchi design; interfacial layer; dissimilar sheet welding

\section{INTRODUCTION}

Several incentives are pushing the application of electromagnetic pulse welding (MPW) toward the attention of the manufacturing industry. A first incentive is the drive towards automation and cost reduction, by reducing the amount of manual labour. The MPW process is a highly repeatable process that has a short process time making it uniquely suited for automation. Since no surface preparation is required, it offers many advantages over traditional welding processes. A second incentive is the increasing demand for welds between dissimilar materials, for reducing weight, improving the conductivity or for adding functionality of a product. MPW can be used for welding dissimilar metal combinations, like aluminium-steel, aluminium-copper, copper-stainless steel, etc... A third and last incentive is the economic climate with increasing energy costs and more taxation on pollution and different kinds of waste. MPW offers an answer to these demands, due to its inherent characteristics: only a small amount of energy is required for creating the weld compared to fusion welding, no filler materials are required and no pre-treatment of the surfaces is required.

Information regarding the influence of the process (eg. the energy input) and geometrical parameters on the weld quality of joined sheet metals is limited. Experimental research on Cu-Al sheets joined by MPW was performed in order to investigate the underlying correlations. The weld quality can be evaluated based on several parameters, like the weld length, the thickness of the intermetallic layer, the lap shear strength, etc.

Regarding the thickness of the intermetallic layer and other parameters defining the interfacial layer, there are several conflicting standpoints. On the one hand, there are authors who claim that an interface with a small intermetallic layer (thickness $<5 \mu \mathrm{m}$ ) and a straight interface are most desired, since a wavy interface is associated with localised melting pockets. Moreover, the formed intermetallic phases can be brittle [1]. Others state that a wavy interface is required to achieve a high weld strength because of mechanical interlocking [2]. A third standpoint claims that there exists a critical width of the intermetallic compounds at which the bond strength is sharply reduced and a transition of ductile to brittle behaviour is observed [3].

\section{PROCESS PRINCIPE OF ELECTROMAGNETIC PULSE SHEET WELDING}

Electromagnetic pulse welding is an impact welding process. In impact welding, a sheet is accelerated over a small distance, called the stand-off distance, up to a velocity of a few hundred $\mathrm{m} / \mathrm{s}$. Then a collision with a rigidly fixed parent plate is created, and a sound weld will be formed on the condition that the attained velocity is high enough and that the angle between the colliding plates is within a certain range. A third condition is the formation of a so-called jet. This shock wave will clean the surfaces of both colliding plates, removing oxides and other contaminations.

The acceleration of the flyer sheet can be actuated by different energy sources: explosives, magnetic fields or even lasers. In case a pulsed magnetic field is used, the process is termed electromagnetic pulse welding. The acceleration by magnetic fields consists of two main steps. First, a large capacitor bank is charged, these banks can typically contain up to $50-100 \mathrm{~kJ}$ of energy. The energy of the capacitor bank $(E)$ can be regulated by the charging voltage $(V)$ as indicated by equation 1 . 


$$
E=C V^{2}
$$

Once the capacitor bank is charged, the energy is discharged through a dedicated coil with the aid of high current switches. A damped sinusoidal current is generated in the circuit, which can be calculated based on the principles of the equivalent RLC circuit. This damped AC current typically has a frequency between 10 and $50 \mathrm{kHz}$ and an amplitude of 50 up to $800 \mathrm{kA}$. It is essential for the entire machine to have a low conductive load in order to enable these high currents.

The sheet welding setup can be simplified by perceiving the coil and sheet as two parallel conductors. If these are both carrying a current with an opposite direction, then Ampere's law states that these two conductors will repel each other. Besides this, Lenz's law states that an induced current flows in the opposite direction such that it opposes the magnetic field which produced it. The combination of the aforementioned laws results in the following: a high transient current is running through the coil. This induces a transient current with an opposite direction in the flyer plate, that is positioned on top of the coil with an isolator sheet in between. These currents will repel each other and as a consequence the flyer plate will be accelerated away from the coil (see Figure 1).

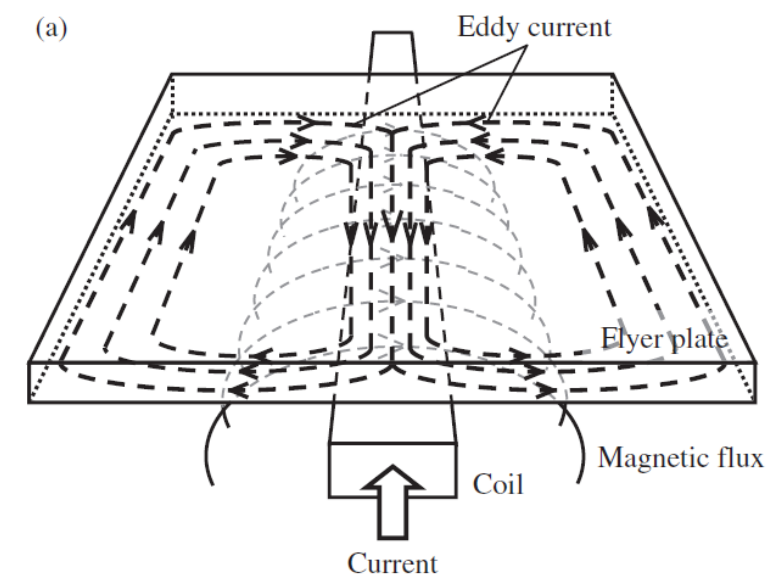

Figure 1. Schematic illustration of the eddy currents in a flat coil set-up [4].

The effect of four parameters on the weld quality was investigated:

- Overlap distance between the sheet and the actuator: defined as the horizontal distance that the flyer sheet overlaps the actuator/coil. The overlap can vary between 0 and $10 \mathrm{~mm}$ (width of the actuator). Overlap values of less than $5 \mathrm{~mm}$ aren't used for practical purposes.

- Free length: defined as the horizontal distance between the extremity of the flyer sheet and the position of the spacer (see Figure 2). This length affects the point where bending of the flyer sheet occurs during the process and also affects the impact angle.

- Stand-off distance: defined as the distance between the flyer and the parent sheet. This distance influences the final impact velocity of the flyer sheet. This parameter also influences the impact angle.

- Energy: depending on the amount of energy that is stored in the capacitors, the current that will discharge through the coil will vary.

- (Frequency): although this parameter is not directly controlled, the coupled inductance of the setup will vary if the geometrical parameters are varied, which influences the discharge current frequency, defined by the equivalent RLC circuit. 


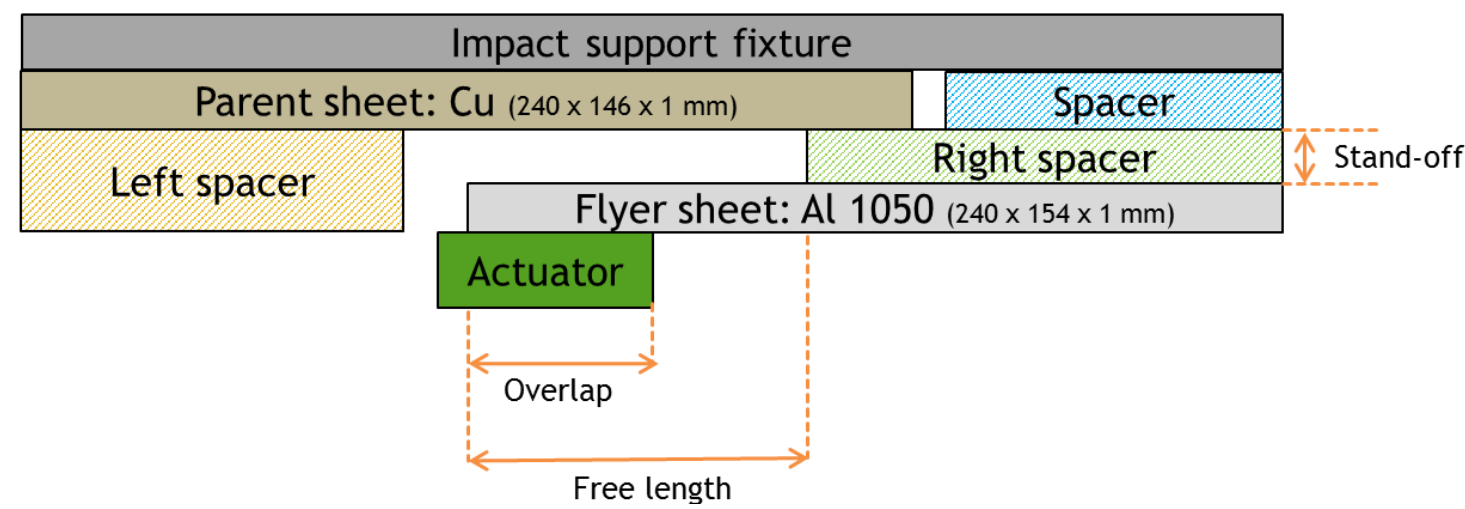

Figure 2. Illustration of the various geometrical parameters that were varied in the sheet welding experiments.

The MPW equipment that was used is a Pulsar system, type MPW 50/25, combined with a coil produced by the company Poynting. Their properties can be found in Table 1.

Table 1. Properties of the coil and MPW equipment

\begin{tabular}{|l|l|l|l|}
\hline \multicolumn{2}{|c|}{ Pulsar MPW 50/25 } & \multicolumn{2}{c|}{ Poynting SMU fvwd 150-1/10 } \\
\hline Maximal charging voltage & $25 \mathrm{kV}$ & $\begin{array}{l}\text { Maximum permitted current } \\
\text { (at reduced lifetime) }\end{array}$ & $280 \mathrm{kA}(400 \mathrm{kA})$ \\
\hline Maximal storage energy & $50 \mathrm{~kJ}$ & Maximum permitted voltage & $20 \mathrm{kV}$ \\
\hline Maximal pulse energy & $40 \mathrm{~kJ}(22.36 \mathrm{kV})$ & Dimensions & $340 \times 230 \mathrm{~mm}$ \\
\hline Maximal pulse current & $500 \mathrm{kA}$ & Main coil conductor length & $240 \mathrm{~mm}$ \\
\hline Voltage-energy ration & $V=\sqrt{\frac{E}{80}}$ & Main coil conductor width & $10 \mathrm{~mm}$ \\
\hline
\end{tabular}

\section{EXPERIMENTAL METHODOLOGY}

The aluminium base material is Al $1050 \mathrm{H} 14$ and the copper base material is ETP Cu R240. Their properties are displayed in Table 2.

Table 2. Mechanical properties of the sheet metals

\begin{tabular}{|l|c|c|}
\hline & Al 1050 H14 [5] & ETP Copper R240 [6] \\
\hline Density & $2710 \mathrm{~kg} / \mathrm{m}^{3}$ & $8940 \mathrm{~kg} / \mathrm{m}^{3}$ \\
\hline Elastic modulus & $71 \mathrm{GPa}$ & $127 \mathrm{GPa}$ \\
\hline Yield stress & $103 \mathrm{MPa}$ & $180 \mathrm{MPa}$ \\
\hline $\begin{array}{l}\text { Ultimate tensile } \\
\text { strength }\end{array}$ & $115 \mathrm{MPa}$ & $240-300 \mathrm{MPa}$ \\
\hline
\end{tabular}

In order to attain reproducible results, an experimental methodology was consistently followed. This methodology consisted of various aspects explained in the subsequent paragraphs.

Both the $\mathrm{Al}$ and $\mathrm{Cu}$ sheet metal surfaces were cleaned manually by first removing the oxides and other contaminants by scouring with steel wool followed by rinsing with acetone for removing possible greases. For the weld quality analysis, the welded plates were cut into six pieces: three specimens with a width of $45 \mathrm{~mm}$ for tensile testing, one specimen with a width of $5 \mathrm{~mm}$ for metallographic processing, the remaining two 
specimens are the remainders left and right of the welded zone. These last two specimens were peeled manually, hence the weld width could be determined. The sample for metallographic analysis was extracted near the middle of the weld. Various measurements were performed during the metallographic examination: the weld length, number of pores and cracks, the composition and size of the interfacial layer. Some typical results will be discussed.

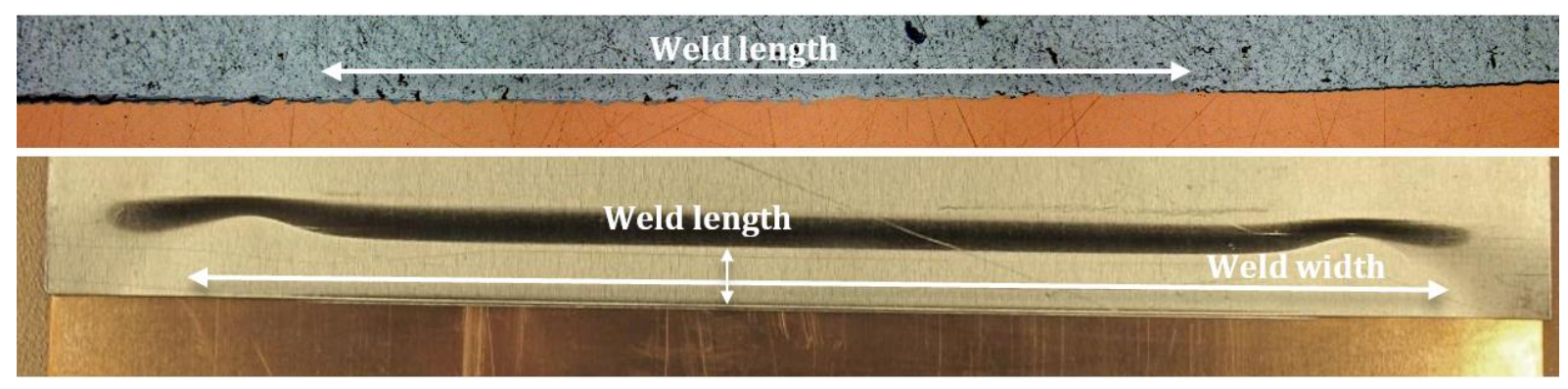

Figure 3. Illustration of the definition of weld length and weld width

After a first series of screening experiments, the range of possible parameter settings was determined, see Table 3. Based on these values, a minimum of 54 experiments would need to be performed for a full factorial design. By making use of experimental design techniques, the amount of experiments was reduced significantly. But this results in a trade-off: a larger number of experiments results in a higher statistical certainty concerning the results. In this investigation, a Taguchi design was used, which has the following advantages. Taguchi designs are typically orthogonal with respect to main effects, but contain either aliased or confounded main effects with 2-way interactions. The main contribution of Taguchi is the robust design, it investigates and detects factors which affect the response value and the variability or dispersion of the response while minimising the variability due to uncontrolled or noise factors [7].

The Taguchi L-18 design type was used. It consists of 18 out of the 54 possible combinations. When performing these experiments, care has to be taken to perform them in a random order. This decreases the chance of making consecutive errors and reduces the possible influence of parameters (nuisance variables) that are not controlled in the experimental design (i.e. temperature, humidity, ...). The 18 experiments were performed only once. A few experiments with unexpected results were performed twice in order to verify their results. Note that MPW is a highly repeatable process and because of this no second run was performed.

Table 3. Values for the input parameters

\begin{tabular}{|c|c|}
\hline Input parameter & Values \\
\hline Energy & $5.5-6.5-7.5 \mathrm{~kJ}$ \\
\hline Stand-off & $1-2-3 \mathrm{~mm}$ \\
\hline Free length & $10-15-20 \mathrm{~mm}$ \\
\hline Overlap & $6-8 \mathrm{~mm}$ \\
\hline
\end{tabular}

\section{RESULTS}

Four main indicators related to the weld quality will be discussed: the weld length, the lap shear strength, the size of the interfacial layer and the thickness reduction of the aluminium sheet.

The influence of the overlap and free length on the weld length is minimal. The two main factors that influence the weld length are the energy and the stand-off. In Figure 4 each point represents one unique combination of input parameters and the blue line connects the averages for a fixed input parameter value. It is clear that a higher amount of energy results in a longer weld length and a smaller dependency on the other input parameters is notable for a high amount of energy. Note that even higher energy inputs are likely to result in a reduced weld length due to large interfacial debonding. 
What concerns the stand-off distance, the largest (average) weld length occurs for a value of $2 \mathrm{~mm}$. Note that it is likely that this is not the optimum value. This relation between the stand-off and weld length can be explained physically: the flyer plate is accelerated away from the coil. The further away from the coil, the smaller the magnetic force, and moreover the current decreases after the first maximum. Because of the combination of these two effects, an optimum stand-off distance exists. For a standoff distance larger than the optimum value, the flyer sheet is already decelerating at the time of impact.
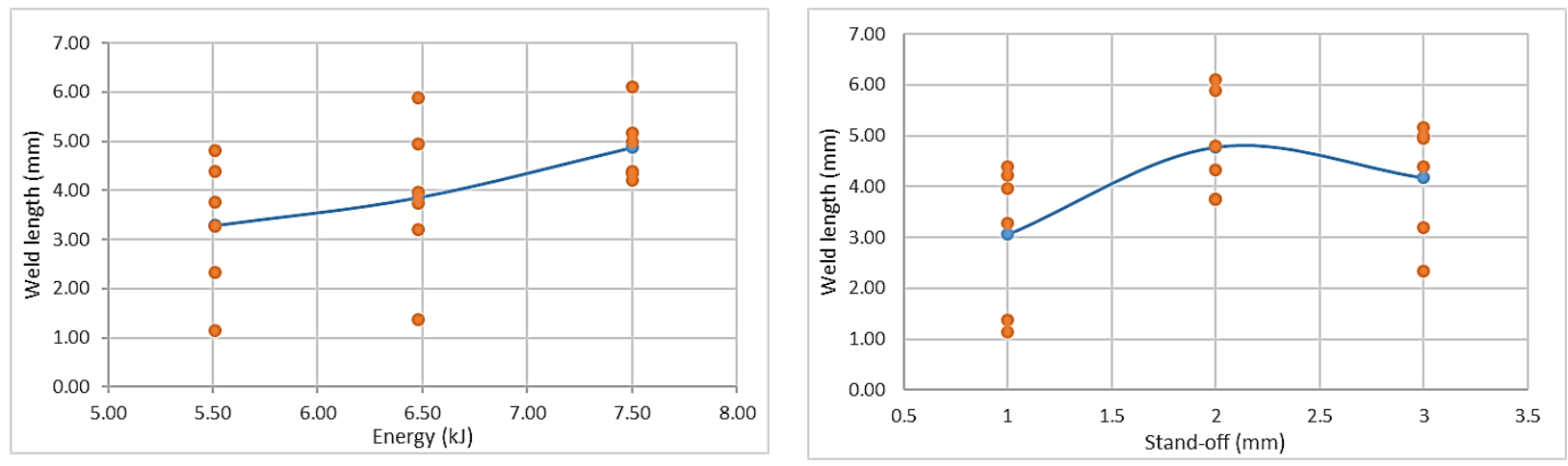

Figure 4. Graphs correlating the weld length and the energy and stand-off respectively

A second indicator for the weld quality is the lap shear strength. It was found that the lap shear strength depends on the aluminium sheet thickness next to the welded zone. Figure 5 shows a linear correlation between the weld strength and the minimum aluminium sheet thickness. This reduction of the aluminium flyer sheet thickness occurred at the left edge of the right spacer (see Figure 2). No influence of the other parameters could be found, even when the weld length was relatively small (around $1 \mathrm{~mm}$ ).
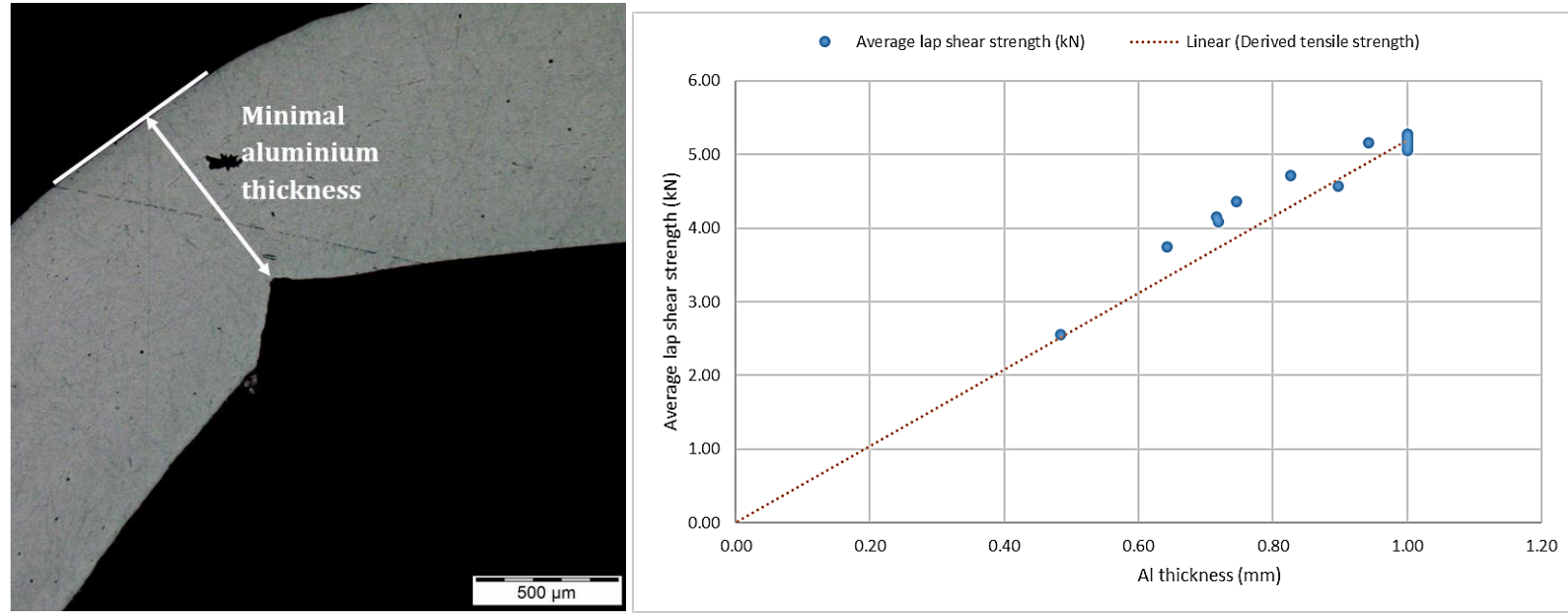

Figure 5. Left: Illustration of the parameter referred to as "minimal aluminium sheet thickness".

Right: Correlation between the lap shear strength and the minimal aluminium sheet thickness. The dotted line represents the theoretical lap shear strength based on the aluminium sheet thickness and the ultimate tensile strength of Al $1050 \mathrm{H} 14$.

The influence of the energy and the overlap on the minimal aluminium sheet thickness is negligible, this in contradiction with the influence of the stand-off distance and the free length. A higher stand-off can result in a large reduction of the aluminium sheet thickness, whereas a low free length always results in a reduced aluminium thickness (Figure 6). A strong interaction was suspected between the two parameters, as can be explained geometrically. For a small free length value, i.e. $10 \mathrm{~mm}$, a large plastic deformation of the flyer sheet takes place at the spacer edge, especially for a big overlap distance of the flyer sheet and the actuator. The worst case is the combination of a large stand-off distance of $3 \mathrm{~mm}$ and an overlap between flyer sheet and actuator of $10 \mathrm{~mm}$. In this case, the aluminium sheet experiences a strong shear effect at the spacer edge.

This suspected interaction is confirmed by Figure 7 and Error! Reference source not found.. Note that the values of the combined parameter (CP) have no practical meaning. It can be concluded that the combination 
of a high stand-off (SO) distance and small free length (FL) results in a large reduction of the aluminium sheet thickness.

$$
(4-\mathrm{SO}) * 5+\mathrm{FL}=\mathrm{CP}
$$
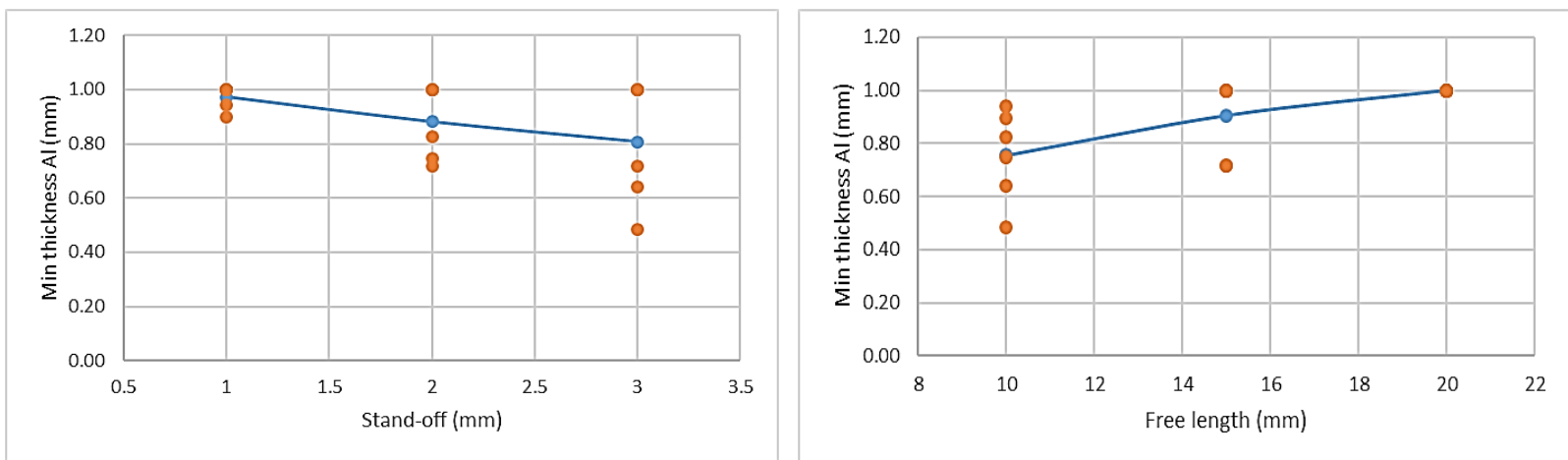

Figure 6: Correlation between the minimal aluminium sheet thickness and the stand-off distance (left) and the free length (right)

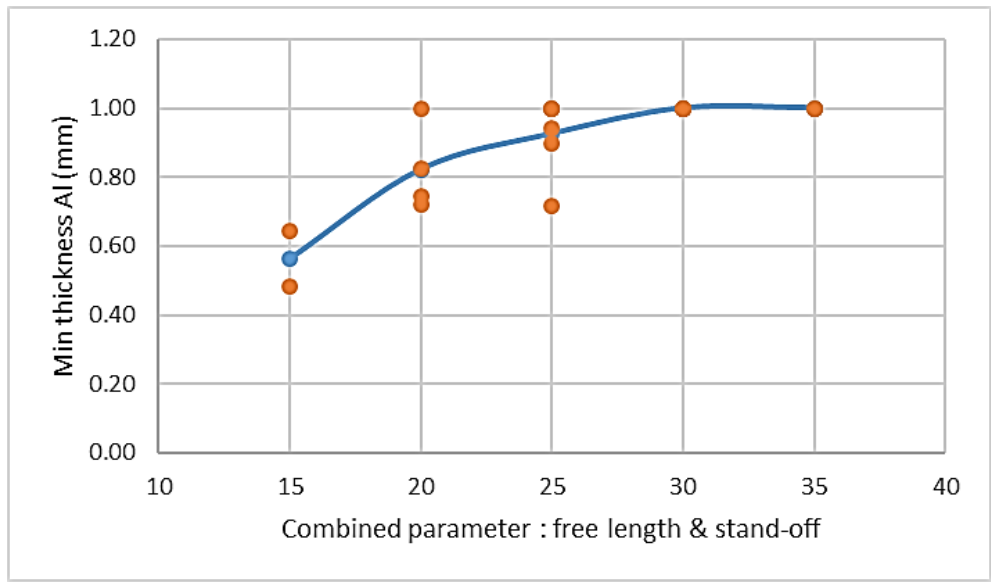

Figure 7: Correlation between the minimal aluminium sheet thickness and a parameter that combines both the free length and the stand-off distance with each a relative weight of $50 \%$

The last indicator for the weld quality is the thickness of the interfacial layer. This quantity was determined by measuring the thickness of the interfacial layer for one out of every three "waves"(Figure 8). The average of these measurements was calculated and then multiplied by the percentage of the length where an intermetallic layer was visibly present with respect to the total weld length.

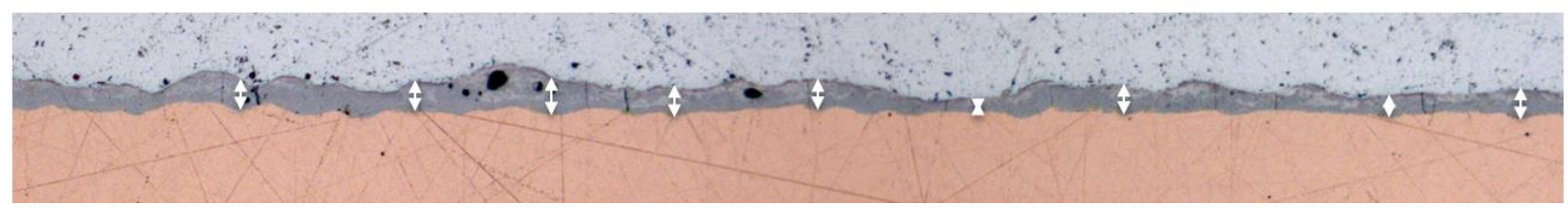

Figure 8: Illustration of the measurement principle for determining the height of the interfacial layer.

Several input parameters affect the thickness of the intermetallic layer, though the relationships are less pronounced than initially perceived. The first and most pronounced influence is from the input energy (Figure 9). If the latter increases, the thickness of the intermetallic layer also increases. A $45 \%$ increase of the energy results in a $100 \%$ increase of the average thickness of the interfacial layer. The effect of the stand-off distance is less pronounced; a maximum is achieved for a stand-off equal to $2 \mathrm{~mm}$. If the two outliers for a stand-off distance equal to $2 \mathrm{~mm}$ would not be present, this parameter wouldn't even be influential (see Figure 9, top right). Further experiments are required in order to confirm the aforementioned correlation. 
The free length has no apparent direct effect on the average thickness of the intermetallic layer, however for a free length of $10 \mathrm{~mm}$, the spread caused due to changing the other input parameters is significantly lower.

One particular combination of the overlap and the stand-off, respectively 8 and $2 \mathrm{~mm}$, results in a significant increase of the average thickness of the interfacial layer. This effect is most pronounced on the graph correlating the energy with the average thickness of the interfacial layer (see Figure 9 ,top right). The maximum thickness for each separate energy level are a combination of the aforementioned input parameter combination.
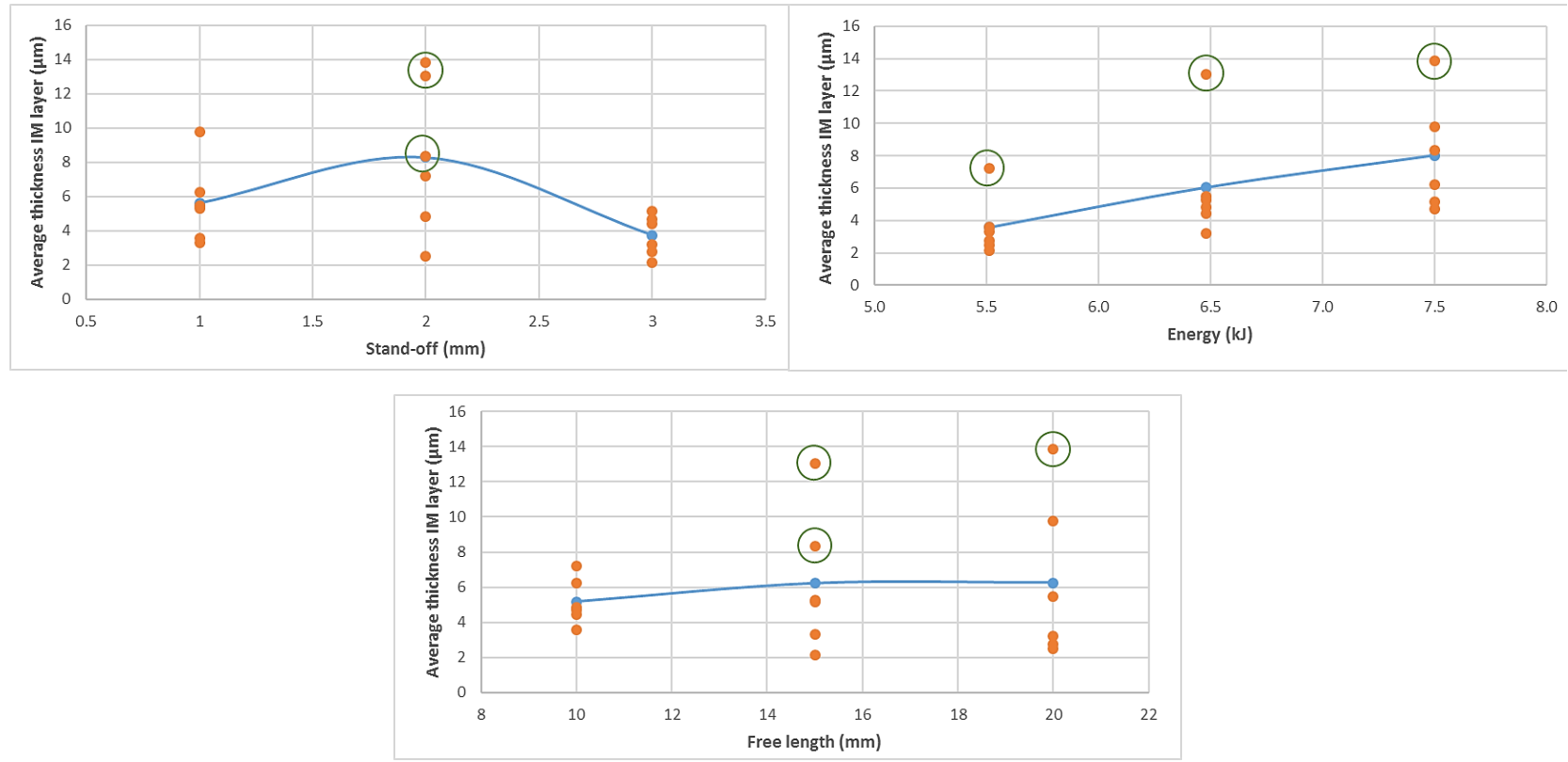

Figure 9: Correlation between the average height of the interfacial layer and the energy, stand-off and free length respectively

\section{CONCLUSIONS}

Aluminium to copper sheets are joined by the electromagnetic pulse welding technology at different process conditions. The following conclusions can be drawn from the present experimental study:

- Most welds were far stronger than the base material.

- The lap shear strength is dependent on the aluminium sheet thickness next to the welded zone. The influence of the energy and the overlap on the minimal aluminium sheet thickness is negligible, this in contradiction with the influence of the stand-off distance and the free length.

- The thickness of the intermetallic layer at the weld interface is mostly influenced by the input energy. The effect of the stand-off distance is less pronounced; a maximum is achieved for a stand-off equal to $2 \mathrm{~mm}$.

- The influence of the overlap and free length on the weld length is minimal. The two main factors that influence the weld length are the energy and the stand-off.

The investigation revealed the important relations between the input parameters and indicators for the weld quality. Armed with this information, it is possible to perform an optimisation for individual or multiple output parameters.

\section{NOMENCLATURE (OPTIONAL)}

$\begin{array}{lll}\text { E } & \text { energy } & \mathrm{J} \\ \text { C } & \text { capacity } & \mathrm{F} \\ \mathrm{V} & \text { voltage } & \mathrm{V} \\ \text { SO } & \text { Stand-off } & \mathrm{mm} \\ \text { FL } & \text { Free length } & \mathrm{mm} \\ \mathrm{CP} & \text { Combined parameter } & /\end{array}$




\section{ACKNOWLEDGEMENTS}

The authors would like to acknowledge the support of the technical staff of the Laboratory Soete and the Belgian Welding Institute.

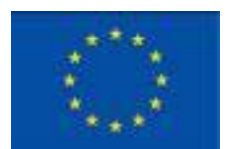

This project has received funding from the European Union's Horizon 2020 research and innovation programme under grant agreement No. H2020-FoF-2014-677660 - JOIN-EM.

\section{REFERENCES}

1. Beyer, E., et al., Insights into intermetallic phases on pulse welded dissimilar metal joints. 2010.

2. Marya, M., S. Marya, and D. Priem, On the characteristics of electromagnetic welds between aluminium and other metals and alloys. Welding in the World, 2005. 49(5-6): p. 74-84.

3. Abbasi, M., A.K. Taheri, and M.T. Salehi, Growth rate of intermetallic compounds in Al/Cu bimetal produced by cold roll welding process. Journal of Alloys and Compounds, 2001. 319(1-2): p. 233241.

4. Watanabe, M. and S. Kumai, High-Speed Deformation and Collision Behavior of Pure Aluminum Plates in Magnetic Pulse Welding. Materials Transactions, 2009. 50(8): p. 2035-2042.

5. Aalco, Specifications 1050A H14 Sheet. 2015.

6. Aurubis, Technical datasheet Cu-ETP. 2005.

7. Pankratz, P.C., Understanding Industrial Designed Experiments. Technometrics, 1992. 34(4): p. $492-$ 492. 